\title{
Genetic redundancy in the catabolism of methylated amines in the yeast Scheffersomyces stipitis
}

\author{
Tomas Linder $\mathbb{D}$
}

Received: 6 August 2017 / Accepted: 12 October 2017 / Published online: 30 October 2017

(C) The Author(s) 2017. This article is an open access publication

\begin{abstract}
The catabolism of choline as a source of nitrogen in budding yeasts is thought to proceed via the intermediates trimethylamine, dimethylamine and methylamine before the release of ammonia. The present study investigated the utilisation of choline and its downstream intermediates as nitrogen sources in the yeast Scheffersomyces stipitis using a reverse genetics approach. Six genes (AMO1, AMO2, SFA1, FGH1, PICST_49761, PICST_63000) that have previously been predicted to be directly or indirectly involved in the catabolism of methylated amines were individually deleted. The growth of each deletion mutant was assayed on minimal media with methylamine, dimethylamine, trimethylamine or choline as the sole nitrogen source. The two amine oxidaseencoding genes $A M O 1$ and $A M O 2$ appeared to be functionally redundant for growth on methylated amines as both deletion mutants displayed growth on all nitrogen sources tested. However, deletion of $A M O 1$ resulted in a pronounced growth lag on all four methylated amines while deletion of AMO2 only caused a growth lag when methylamine was the sole nitrogen source. The glutathione-dependent
\end{abstract}

Electronic supplementary material The online version of this article (doi:10.1007/s10482-017-0963-y) contains supplementary material, which is available to authorized users.

T. Linder $(\bowtie)$

Department of Molecular Sciences, Swedish University of Agricultural Sciences, Box 7015, 75007 Uppsala, Sweden e-mail: tomas.linder@slu.se formaldehyde dehydrogenase-encoding gene SFAI was found to be absolutely essential for growth on all methylated amines tested while deletion of the $S$ formylglutathione hydrolase gene $F G H 1$ caused a pronounced growth lag on dimethylamine, trimethylamine and choline. The putative cytochrome P450 monooxygenase-encoding genes PICST_49761 and PICST_63000 were considered likely candidates for demethylation of di- and trimethylamine but produced no discernable phenotype on any of the tested nitrogen sources when deleted. This study revealed notable instances of genetic redundancies in the choline catabolic pathway, which are discussed.

Keywords Amine $\cdot$ Metabolism $\cdot$ Reverse genetics . Yeast
Abbreviations
CYP Cytochrome P450 monooxygenase
IGR Intergenic region
NHEJ Non-homologous end-joining pathway
PTS Peroxisomal targeting signal

\section{Introduction}

Amines are organic nitrogen compounds common in the environment and consequently most microorganisms have evolved pathways to assimilate amines as a source of metabolic nitrogen. The ability to assimilate 
amines as a nitrogen source is nearly universal among budding yeasts (phylum Ascomycota, sub-phylum Saccharomycotina) but does not occur in the common model system Saccharomyces cerevisiae (van der Walt 1962; van Dijken and Bos 1981; Linder 2014). Due to the limited research hitherto conducted on nonSa. cerevisiae yeasts, our understanding of the genetics governing the assimilation of amines in budding yeasts remains rudimentary.

Previous biochemical studies on budding yeasts capable of assimilating amines have shown that the deamination of primary amines $\left(\mathrm{RCH}_{2} \mathrm{NH}_{2}\right)$ is catalysed by copper-containing amine oxidases (EC 1.4.3.6) to release ammonia, hydrogen peroxide and the corresponding alkylaldehyde (RCHO). Most budding yeasts appear to possess two types of amine oxidases, which are commonly referred to as methylamine oxidase and benzylamine oxidase, respectively (Haywood and Large 1981; Green et al. 1982). Methylamine oxidase (encoded by the AMO1 gene) appears to have higher affinity towards short-chain aliphatic amines while benzylamine oxidase (encoded by the $A M O 2$ gene) has higher affinity towards amines with longer and bulkier side-chains (Haywood and Large 1981). The Amo1 methylamine oxidase contains an $\mathrm{N}$-terminal type 2 peroxisomal targeting signal (PTS2) composed of the nonapeptide motif RLXXXXX ${ }^{\mathrm{H}} / \mathrm{Q} \mathrm{L}$ and localises to the peroxisome (Zwart et al. 1980; Faber et al. 1994) while the Amo2 benzylamine oxidase is thought to be localised to the cytosol.

Secondary $\left[\left(\mathrm{RCH}_{2}\right)_{2} \mathrm{NH}\right]$ and tertiary $\left[\left(\mathrm{RCH}_{2}\right)_{3} \mathrm{~N}\right]$ amines can also be assimilated by some yeasts (van Dijken and Bos 1981; Linder 2014) but the identity of the enzymes involved in the de-alkylation of these substrates into primary amines have yet to be established. Choline is one of the few quaternary (tetraalkylated) amines that is known to be de-alkylated and subsequently assimilated by budding yeasts (van Dijken and Bos 1981; Linder 2014). The catabolism of choline is thought to involve four sequential dealkylation steps via the intermediates trimethylamine, dimethylamine and methylamine before ammonia is released by amine oxidase (Zwart et al. 1980, 1983; Fig. 1). Understanding of the genetics surrounding this pathway remains incomplete. A putative choline monooxygenase encoded by the CMOI gene is thought to be responsible for the first de-alkylation step to produce trimethylamine and glycolaldehyde (Mori et al. 1988; Linder 2014). The yeast Cmo1 choline<smiles>C[N+](C)(C)CCO</smiles>

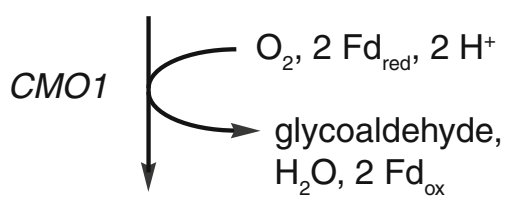

trimethylamine<smiles>CN(C)C</smiles>

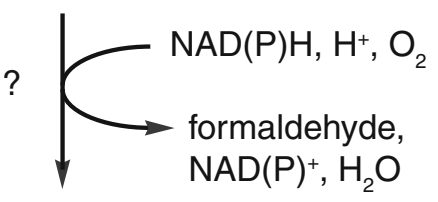

dimethylamine<smiles>CNC</smiles>

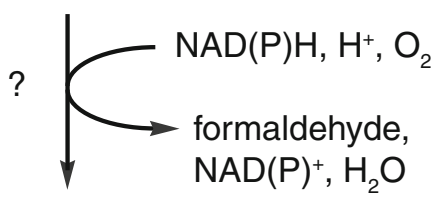

methylamine<smiles>CN</smiles><smiles>COC(C)(C)C(CO)C(=O)OC(C)(C)C</smiles>

Fig. 1 The proposed pathway for choline assimilation in budding yeasts

protein has not yet been biochemically characterised but the homologous plant protein (which converts choline into betaine aldehyde in the chloroplast) requires oxygen and ferredoxin (Brouquisse et al. 1989). Biochemical studies of the demethylation of diand trimethylamine indicate that two distinct enzyme activities are involved, which have characteristics typical of cytochrome P450 monooxygenases (Green and Large 1983, 1984; Fattakhova et al. 1991). The 
final demethylation step involves one or both amine oxidases (Haywood and Large 1981).

The present study set out to study the genetics surrounding the catabolism of choline and focused on six genes thought to be directly involved in either the amine de-alkylation steps or linked reactions such as detoxification of the significant amounts of formaldehyde generated by each demethylation reaction. The yeast Scheffersomyces stipitis was selected as a model system for this study as it can assimilate all the predicted intermediates of the choline pathway (Linder 2014).

\section{Materials and methods}

Sc. stipitis integration constructs

Targeting cassettes for AMO1 (PICST_55523), AMO2 (PICST_83878), SFA1 (PICST_29252), FGH1 (PICST_65460), PICST_49761 and PICST_63000 were synthesised de novo by GenScript (NJ, USA) and inserted into either EcoRI/HindIII-cut or EcoRI/ StuI-cut pUC57 (GenBank accession Y14837; Fig. S1a). Each targeting cassette consisted of $500 \mathrm{bp}$ sequence identical to the intergenic region immediately downstream ( $3^{\prime}$ IGR) of the gene to be deleted ("PICST_xxxxx") followed by $500 \mathrm{bp}$ sequence immediately upstream ( $5^{\prime}$ IGR) of the sequence to be deleted (Fig. S1b). The $3^{\prime}$ and $5^{\prime}$ IGR sequences in each targeting cassette were separated by a SwaI recognition site. Each targeting cassette was followed by a short polylinker to enable insertion of a selection marker. The full-length HIS3 gene was amplified from Debaryomyces hansenii CBS 767 genomic DNA with primers DhHIS3 fwd (5' GCG CGC GGA TCC TTT CAC CAG ATG GGA TCT AAT $3^{\prime}$ ) and DhHIS3 rev ( $5^{\prime}$ GCG CGC CTG CAG GCG CGC CAG TCG TAA TGT TTA TAG AAG A $3^{\prime}$ ), digested with BamHI and PstI and inserted into each targeting construct, which had been digested with the appropriate enzymes to produce ends compatible with BamHI (either BamHI or BglII) and PstI (PstI or SbfI). The restriction sites used to insert the DhHIS3 gene into each targeting plasmid are indicated in Fig. S1a. Prior to transformation, the integration plasmids (Fig. S1c) were digested with SwaI to produce linearised integration constructs (Fig. S1d), which were then purified into sterile water using the QIAquick PCR purification kit (Qiagen).

Yeast transformation

The transgenic yeast strains used in this study are listed in Table 1. The transformation methodology has been described previously (Linder 2014). The Sc. stipitis SF1 strain, which lacks a functional nonhomologous end-joining DNA repair pathway (Maassen et al. 2008), was kindly provided by Prof Ulrich Klinner (Aachen University, Germany). Correct chromosomal integration and the deletion of each targeted gene ("PICST_xxxxx") was confirmed by PCR analysis of purified genomic DNA from each deletion strain (Fig. S2) using the primers listed in Table S1.

Nitrogen utilisation assays

Sodium L-glutamate and the hydrochloride salts of methylamine, dimethylamine, trimethylamine and choline were purchased from Sigma Aldrich. A reduced sulfur/nitrogen-limited glucose medium (RSNLD) was used for assaying growth on individual amines. RSNLD medium is composed of $1.7 \mathrm{~g}$ Difco yeast nitrogen base without amino acids or ammonium sulfate $1^{-1}$ (Becton, Dickinson and Company) and $20 \mathrm{~g}$ glucose $1^{-1}$. Prior to the nitrogen utilisation assay, individual yeast strains were pre-cultured in $3 \mathrm{ml}$ minimal glucose medium (MMD) consisting of $6.7 \mathrm{~g}$ Difco yeast nitrogen base without amino acids $1^{-1}$ (Becton, Dickinson and Company) and $20 \mathrm{~g}$ glucose $1^{-1}$. Pre-cultures were washed twice in RSNLD before being re-suspended in $2.97 \mathrm{ml}$ RSNLD to a final $\mathrm{OD}_{600}$ of 0.005 in a $50-\mathrm{ml}$ tube. Individual nitrogen sources were added as $30 \mu \mathrm{l}$ of a $1 \mathrm{M}$ stock solution, making a final concentration of $10 \mathrm{mM}$. A non-supplemented sample with $30 \mu \mathrm{l}$ deionised water was used as a control. Chloramphenicol (final concentration $15 \mathrm{mg} \mathrm{l}^{-1}$ ) was included to prevent bacterial contamination. Samples were incubated at $30{ }^{\circ} \mathrm{C}$ in a rotary shaker set to $200 \mathrm{rpm}$ with $\mathrm{OD}_{600}$ measurements after 6,12 and 18 days. $\mathrm{OD}_{600}$ measurements were carried out with a $1 \mathrm{~cm}$ path length using an Ultrospec 1100 pro spectrophotometer (GE Healthcare). Each growth assay was performed in triplicate with each biological replicate using a separate pre-culture. The yeast strain Ogataea parapolymorpha CBS 11895 was purchased from 
Table 1 Genetically modified Sc. stipitis strains used in this study

\begin{tabular}{lll}
\hline Strain number & Relevant genotype & Source \\
\hline SF1 & his3-1 trp5-10 YKU80::ScTRP5 & U. Klinner (Maassen et al. 2008) \\
TLSS001 & HIS3 trp5-10 YKU80::ScTRP5 & Linder (2014) \\
TLSS005 & his3-1 trp5-10 YKU80::ScTRP5 PICST_55523::pUC57-DhHIS3 & This study \\
TLSS006 & his3-1 trp5-10 YKU80::ScTRP5 PICST_83878::pUC57-DhHIS3 & This study \\
TLSS007 & his3-1 trp5-10 YKU80::ScTRP5 PICST_29252::pUC57-DhHIS3 & This study \\
TLSS008 & his3-1 trp5-10 YKU80::ScTRP5 PICST_65460::pUC57-DhHIS3 & This study \\
TLSS009 & his3-1 trp5-10 YKU80::ScTRP5 PICST_49761::pUC57-DhHIS3 & This study \\
TLSS010 & his3-1 trp5-10 YKU80::ScTRP5 PICST_63000::pUC57-DhHIS3 & This study \\
\hline
\end{tabular}

Centraalbureau voor Schimmelcultures (Utrecht, the Netherlands).

Sequence alignment and phylogenetic analysis

All BLASTP and TBLASTN searches applied an expect value cut-off of $10^{-5}$ with the low-complexity region filter enabled. Protein sequences were aligned in MAFFT (Katoh et al. 2005; http://mafft.cbrc.jp/ alignment/server/index.html). Selection of sequence positions suitable for phylogenetic analysis was carried out in GBlocks (Castresana 2000; http://molevol.ibmb. csic.es/Gblocks_server/). The resulting amino acid positions were then used to construct a neighbourjoining tree in MEGA v. 6 (Tamura et al. 2013) using a JTT amino acid substitution model. Branch support was tested using 10,000 bootstrap replicates. Any nodes with bootstrap values equal or less than 50 were collapsed. The consensus trees were visualised in FigTree v.1.0 (http://tree.bio.ed.ac.uk/software/figtree/).

\section{Results and discussion}

Previous studies on the catabolism of choline and its putative downstream intermediates in budding yeasts have mainly employed biochemical and cell imaging methods (for example Zwart et al. 1980; Haywood and Large 1981; Zwart et al. 1983). Parallel analysis of gene deletion phenotypes under specific growth conditions (phenomics) is a useful tool for the identification of functional redundancies and genetic associations. Measuring cell growth in batch culture is particularly informative as it is possible to resolve the individual dynamic components of the typical sigmoidal cellular growth curve such as growth lag, growth rate and growth efficiency (Warringer et al. 2003). Very little reverse genetics have been done in Sc. stipitis to date due to a scarcity of selection markers and the low targeting frequency of integration constructs due to the domination of the non-homologous end-joining (NHEJ) pathway over homologous recombination. However, the development of a $S c$. stipitis strain auxotrophic for histidine that also lacks a functional NHEJ pathway through deletion of the YKU80 gene has now enabled reverse genetic investigations in this yeast (Maassen et al. 2008). Targeting cassettes for each of the six genes to be deleted in this study (AMO1, AMO2, SFA1, FGH1, PICST_49761, PICST_63000) were synthesised de novo to contain $500 \mathrm{bp}$ of flanking sequences adjacent to the deleted region (Fig. S1a). The D. hansenii HIS3 gene was inserted into each integration construct to enable selection for positive transformants on minimal medium lacking histidine (Fig. S1b-c). A Sc. stipitis $\triangle y k u 80$ strain with a regenerated endogenous HIS3 locus (Linder 2014) was used as a wildtype control in all growth assays.

The first two genes to be investigated were the two amine oxidase-encoding genes AMO1 and AMO2. Each deletion mutant was cultivated in nitrogenlimited medium supplemented with either sodium Lglutamate, methylamine, dimethylamine, trimethylamine or choline. Growth was monitored every 6 days up until 18 days after initiation of the growth assay (Fig. 2). Both mutants displayed strong growth on sodium L-glutamate equivalent to the wildtype control. Interestingly both mutants displayed an identical growth lag on methylamine with strong growth detectable only after the 12-day time-point. In 


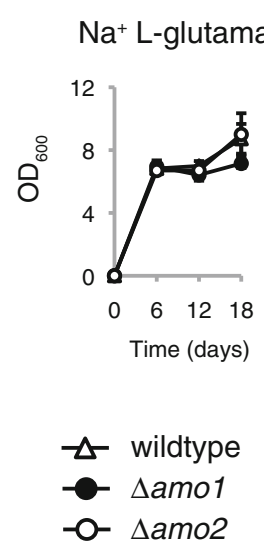

Fig. 2 The requirement for amine oxidase genes $A M O 1$ and $A M O 2$ for the utilisation of methylated amines as sole nitrogen sources. Sc. stipitis strains TLSS001 (wildtype control),

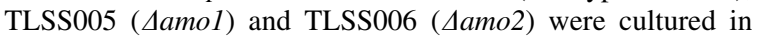
$3 \mathrm{ml}$ RSNLD medium supplemented with $10 \mathrm{mM}$ of the

addition, both mutant strains displayed notably higher cell densities at day 12 and 18 on methylamine compared to the wildtype control. The reason for this effect was not immediately obvious but one possibility could be that the initial lag phase indirectly enabled more efficient energy metabolism once growth was initiated. The $\triangle a m o 2$ strain was indistinguishable from the wildtype control on dimethylamine, trimethylamine and choline, which indicated that AMO2 was entirely dispensable for normal growth on these substrates. The $\Delta$ amol strain displayed a characteristic growth lag on dimethylamine, trimethylamine and choline, which indicated that $A M O 1$ was only partially dispensable for growth on these substrates.

It was notable that the $\triangle a m o 2$ strain had no obvious growth defect on dimethylamine, trimethylamine or choline yet displayed a pronounced lag in growth on methylamine, which is a down-stream intermediate of the three other amines. One possibility is that when methylamine is supplied as an external nitrogen source, the activities of $A M O 1$ and $A M O 2$ must cooperate to achieve maximum growth while $A M O 2$ does not play a significant role in the deamination of methylamine generated intracellularly through demethylation of di- and trimethylamine.

Each of the three demethylation steps in the choline assimilation pathway produces an equimolar amount of formaldehyde to the amount of methylated amine (Fig. 1). The assimilation of methylated amines indicated nitrogen source (initial $\mathrm{OD}_{600}$ 0.005). Samples were incubated in a shaker set at $30{ }^{\circ} \mathrm{C}, 200$ r.p.m., and $\mathrm{OD}_{600}$ was measured after 6, 12 and 18 days. Growth assays were performed in triplicate with error bars indicating one standard deviation

therefore generates significant amounts of highly reactive formaldehyde that must be appropriately metabolised by the cell to ensure viability. The principal pathway for the detoxification of formaldehyde in yeast is the cyclic glutathione-dependent formaldehyde oxidation pathway (Fig. 3a). Formaldehyde enters the pathway through a non-enzymatic reaction with glutathione to form $S$-hydroxymethylglutathione. $S$-hydroxymethylglutathione is then converted into $S$-formylglutathione by glutathionedependent formaldehyde dehydrogenase, which is encoded by the SFAl gene in yeast (Sasnauskas et al. 1992). Glutathione is then regenerated through the action of $S$-formylglutathione hydrolase (encoded by the FGHl gene) to release formic acid (Degrassi et al. 1999; Yurimoto et al. 2003). Formic acid is subsequently oxidised into carbon dioxide by formate dehydrogenase, which is encoded by the $F D H 1$ gene (Allen and Holbrook 1995). The deletion of either SFA1 or FGHI was therefore expected to cause rapid glutathione depletion and ultimately cell death under conditions of high levels of formaldehyde production, such as cultivation on medium where methylated amines were the sole nitrogen source.

No obvious growth defect with sodium L-glutamate as nitrogen source was observed upon deletion of either SFAl or FGHI in Sc. stipitis (Fig. 3b). As expected, the $\Delta s f a l$ strain did not show any detectable growth when any of the methylated amines were the sole nitrogen source. This is in contrast with 


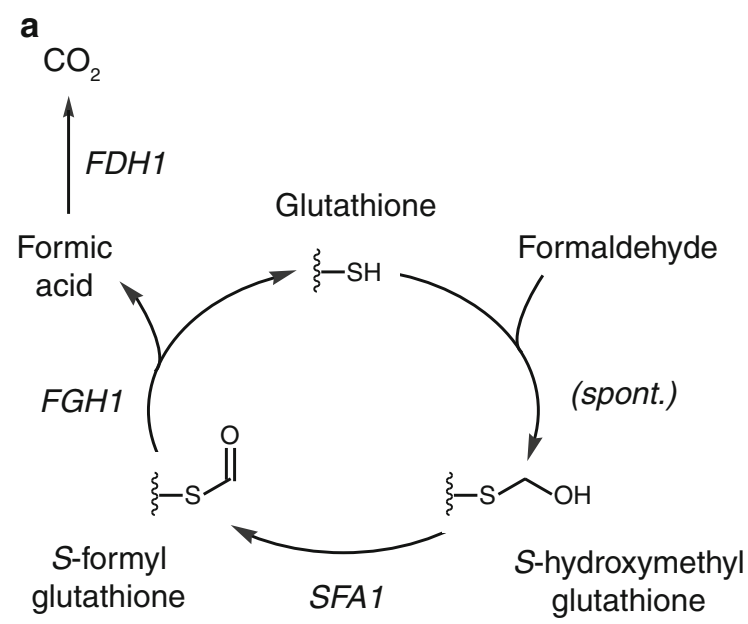

b $\quad \mathrm{Na}^{+}$L-glutamate

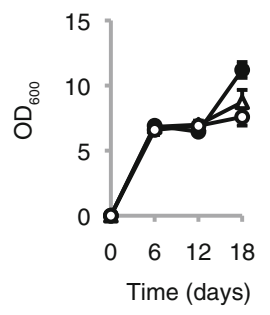

dimethylamine

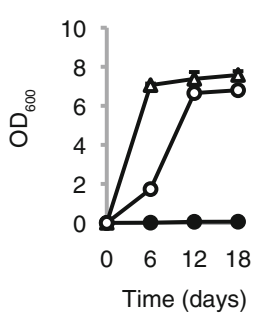

choline

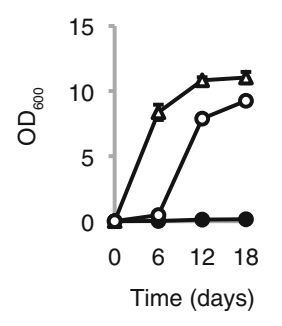

previous work in the methylotrophic yeast Ogataea boidinii where the $\Delta s f a l$ cells displayed weak but
4Fig. 3 The requirement for the glutathione-dependent formaldehyde dehydrogenase gene $S F A 1$ and the $S$-formylglutathione hydrolase gene $F G H 1$ for the utilisation of methylated amines as sole nitrogen sources. a A simplified overview of the glutathione-dependent formaldehyde detoxification pathway. Only the thiol group of glutathione (-SH) is shown. b Sc. stipitis strains TLSS001 (wildtype control), TLSS007 ( $\Delta s f a 1)$ and TLSS008 ( $\Delta f g h l$ ) were cultured in $3 \mathrm{ml}$ RSNLD medium supplemented with $10 \mathrm{mM}$ of the indicated nitrogen source (initial $\mathrm{OD}_{600}$ 0.005). Samples were incubated in a shaker set at $30{ }^{\circ} \mathrm{C}, 200$ r.p.m., and $\mathrm{OD}_{600}$ was measured after 6, 12 and 18 days. Growth assays were performed in triplicate with error bars indicating one standard deviation

detectable growth on both methylamine and choline (Lee et al. 2002). One possibility is that $O$. boidinii possesses a second functionally redundant enzyme capable of converting $S$-hydroxymethylglutathione into $S$-formylglutathione.

Notably the $\Delta f g h l$ strain showed significant growth on all methylated amines and in the case of methylamine the $\Delta f g h l$ strain displayed noticeably higher cell densities than the wildtype control. However, the $\Delta f g h l$ strain displayed a pronounced growth lag on dimethylamine, trimethylamine and choline reminiscent of the growth dynamics observed with the $\Delta a m o l$ strain (Fig. 2). Direct comparison of the growth curves for the $\Delta f g h l$ and $\Delta a m o l$ strains showed a striking overlap in growth dynamics (Fig. S3), which could indicate a genetic link between the two genes. One possibility is that Amo1-dependent demethylation of methylamine derived from dimethylamine, trimethylamine and choline in Sc. stipitis requires Fgh1 while Amo2-dependent demethylation does not. In O. boidinii the Fgh1 protein has been shown to localise both in peroxisomes and the cytosol (Yurimoto et al. 2003). Assuming that the Sc. stipitis Fgh1 protein displays the same localisation pattern as its $O$. boidinii ortholog, the Fgh1 protein would available to both amine oxidase isoenzymes. The fact that $S F A l$ is essential for growth on methylated amines in Sc. stipitis but FGH1 is not would suggest there are additional pathways in this yeast for the regeneration of glutathione from $S$-formylglutathione. Previous studies of the $F G H 1$ gene in $O$. boidinii have shown that deletion of $F G H 1$ in this yeast results in detectable but retarded growth on both methylamine and choline (Yurimoto et al. 2003), which would suggest the existence of a functionally redundant enzyme in this yeast as well. 
The enzymes responsible for demethylation of diand trimethylamine in budding yeasts remain to be identified (Fig. 1). Previous biochemical studies have suggested that cytochrome P450 monooxygenases may be responsible for these reactions (Green and Large 1983, 1984; Fattakhova et al. 1991). A survey of Sc. stipitis genes encoding proteins belonging to the cytochrome P450 (CYP) monooxygenase family was therefore conducted. A total of ten genes in the $S c$. stipitis genome have been assigned to the CYP superfamily (Chen et al. 2014; Fig. S4). Three of the Sc. stipitis CYP genes appeared to be the orthologs of the $S a$. cerevisiae genes DIT2 ( $N$-formyltyrosine oxidase, family CYP56), ERG5 (C-22 sterol desaturase, family CYP61) and ERG11 (lanosterol 14 $\alpha$ demethylase, family CYP51), respectively. The genes ERG5 and ERG11 are involved in the biosynthesis of the essential membrane steroid ergosterol (Turi et al. 1991; Kelly et al. 1995) and both genes have so far been found in all sequenced yeast genomes. The DIT2 gene is involved in the biosynthesis of dityrosine, which is a component of the spore cell wall (Briza et al. 1994). However, the DIT2 gene does not appear to be universally conserved among budding yeasts (data not shown).

The remaining seven CYP-family genes lacked $S a$. cerevisiae counterparts but five of them shared sequence similarity with $n$-alkane monooxygenases and fatty acid w-hydroxylases ( $A L K$, family CYP52), which have been previously described in other yeasts (Sanglard and Loper 1989; Ohkuma et al. 1995; Huang et al. 2014). The two remaining Sc. stipitis CYP genes PICST_49761 and PICST_63000 have previously been assigned to CYP families CYP501 and CYP5217, respectively (Chen et al. 2014). Neither of these two CYP families have been biochemically characterised at the time of writing.

To avoid the necessity of deleting all seven CYP genes, a bioinformatic survey was made of genomes of other budding yeasts to identify species capable of assimilating multi-alkylated amines while simultaneously possessing a smaller repertoire of CYP-family genes than Sc. stipitis. The genome of the yeast Ogataea parapolymorpha was found to contain five CYP-family genes in total (Chen et al. 2014; Fig. S4) as well as a homolog of the CMO1 gene (systematic gene name HPODL_01912), which predicted that this species should be able to assimilate choline and therefore di- and trimethylamine as well. $O$. parapolymorpha was therefore tested for its ability to use trimethylamine as a nitrogen source and was found to display strong growth (Fig. S5a). Phylogenetic analysis of the peptide sequences from the full CYP gene family complement of $O$. parapolymorpha, Sa. cerevisiae and Sc. stipitis identified two distinct clades of unknown function, which contained CYPfamily proteins from both $O$. parapolymorpha and $S c$. stipitis but none from $\mathrm{Sa}$. cerevisiae (Fig. S5b). The first clade contained the gene products of the $O$. parapolymorpha and Sc. stipitis genes HPODL_02874 and PICST_63000, respectively. The bootstrap support for this clade was moderate $(68 \%)$ with the two proteins being $30 \%$ identical and $45 \%$ similar. A recent classification effort of fungal CYP genes placed the HPODL_02874 and PICST_63000 genes in separate CYP families CYP5223 and CYP5217, respectively (Chen et al. 2014). The second clade had strong bootstrap support (97\%) and consisted of the gene products of $O$. parapolymorpha genes HPODL_02307 and HPODL_00882 as well as the gene product of the Sc. stipitis gene PICST_49761. HPODL_02307 formed an internal node with PICST_49761 with $83 \%$ bootstrap support and the protein sequences of the gene products were $37 \%$ identical and $54 \%$ similar. The HPODL_02307 and PICST_49761 genes are currently assigned to the CYP501 family while the HPODL_00882 gene has been placed in the CYP504 family (Chen et al. 2014).

The assumption was made that if CYP-family enzymes were involved in the demethylation of di- and trimethylamine, these enzymes were expected to be shared between $S c$. stipitis and O. parapolymorpha but lacking in Sa. cerevisiae. The only two Sc. stipitis CYP-encoding genes that satisfied this criterion were the two uncharacterised genes PICST_49761 and PICST_63000. The two Sc. stipitis genes PICST_49761 and PICST_63000 were therefore deleted and tested for growth on different nitrogen sources. However, neither deletion mutant displayed any obvious growth defect on methylated amines compared to the wildtype control (Fig. 4). This suggests that PICST_49761 and PICST_63000 do not encode either of the CYP-family enzymes or CYPlike enzymatic activities thought to catalyse the demethylation of di- and trimethylamine. These results suggest that the enzymes responsible for the demethylation of di- and trimethylamines may in fact belong to another enzyme family with biochemical 


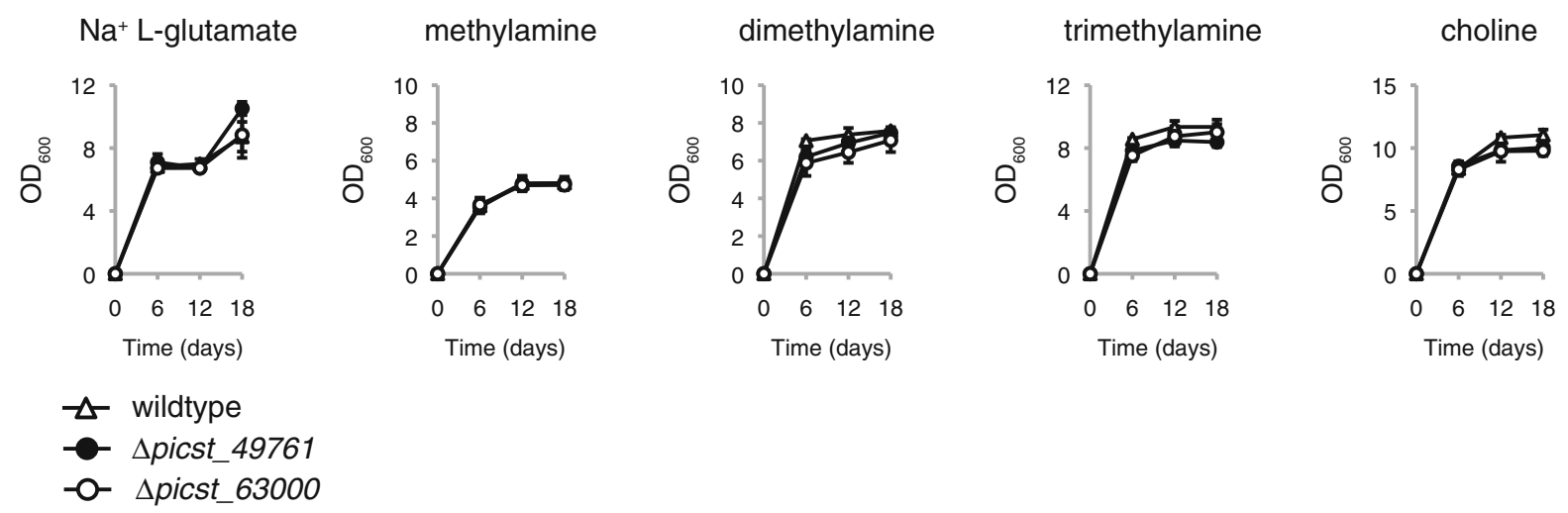

Fig. 4 The requirement for the putative cytochrome P450 (CYP) monooxygenase genes PICST_49761 and PICST_63000 for the utilisation of methylated amines as sole nitrogen sources. Sc. stipitis strains TLSS001 (wildtype control), TLSS009 (4picst_49761) and TLSS010 (4picst_63000) were cultured in $3 \mathrm{ml}$ RSNLD medium supplemented with $10 \mathrm{mM}$ of the

properties similar to that of CYP monooxygenases that had been reported previously (Green and Large 1983, 1984; Fattakhova et al. 1991). Another possibility is that a diversity of pathways exists in different yeasts for the demethylation of di- and trimethylamine, where one or more alternative enzyme families are responsible for these enzymatic steps in $S c$. stipitis while CYP-family enzymes catalyse these reactions in the yeast species studied previously. A third possibility is that there is functional redundancy within the CYP superfamily so that the Sc. stipitis CYP52 family $A L K$-like monooxygenases also possess amine demethylation activity. This could be tested through sequential deletion of all seven members of CYP families CYP52, CYP501 and CYP5217 in Sc. stipitis. However, the current limitation in genetic tools for this $S c$. stipitis makes this approach impractical at present.

In summary, this study demonstrates the substantial gap that remains in functional annotation of genomes from so-called non-conventional yeasts. The common baker's yeast $S a$. cerevisiae has long been the dominant system for the application of reverse genetics to the study of yeast metabolism. However, the limited number of nitrogen substrates assimilated by Sa. cerevisiae makes it unsuited to the study of poorly characterised nitrogen assimilation pathways (Large 1986; Linder 2014). This study focused specifically on the yeast choline catabolic pathway, which has previously been studied using predominantly indicated nitrogen source (initial $\mathrm{OD}_{600}$ 0.005). Samples were incubated in a shaker set at $30{ }^{\circ} \mathrm{C}, 200$ r.p.m., and $\mathrm{OD}_{600}$ was measured after 6, 12 and 18 days. Growth assays were performed in triplicate with error bars indicating one standard deviation

biochemical methods (for example Green and Large 1983, 1984; Mori et al. 1988; Fattakhova et al. 1991). A complementary reverse genetics approach was used in this study and the resulting data can be condensed into three general observations.

The first general observation is that there appeared to be a consistent difference between growth patterns on methylamine versus dimethylamine, trimethylamine and choline in Sc. stipitis. Methylamine was the only methylated amine substrate where a growth defect was observed in a $\triangle a m o 2$ strain (Fig. 2). Conversely, methylamine was the only methylated amine substrate where a growth defect was not observed in a $\Delta f g h l$ strain (Fig. 3b) as well as the only methylated amine substrate where the growth dynamics of the $\Delta a m o l$ and $\Delta f g h l$ strains deviated significantly from each other (Fig. S3). One hypothesis to explain this observation is that cell compartmentalisation distinguishes methylamine provided externally in the growth medium versus methylamine synthesised internally from the demethylation of dimethylamine, trimethylamine and choline (Fig. 5). External methylamine would be expected to enter directly into the cytosol with some portion further transported into the peroxisome. This could explain how both amine oxidase mutants displayed an extended lag before initiating growth (Fig. 2). Internally generated methylamine is expected to originate from the endoplasmic reticulum as this is the reported localisation of di- and trimethylamine 


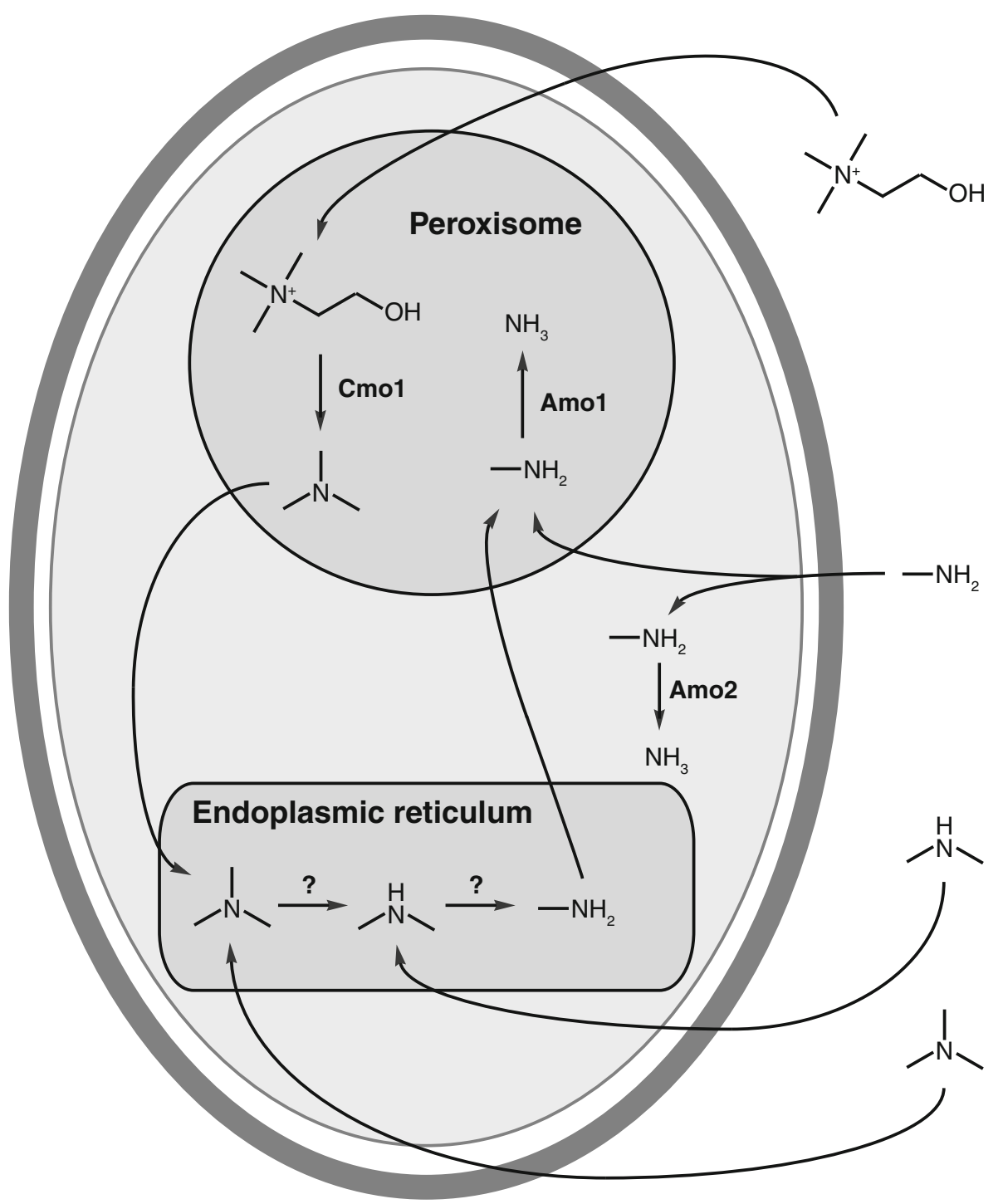

Fig. 5 A model of intracellular compartmentalisation of catabolism of methylated amines in Sc. stipitis based on the data presented in this study as well as previous reports (Zwart et al. 1980, 1983; Green and Large 1984). In the proposed model, extracellular methylamine is demethylated by both Amo1 and Amo2 while methylamine produced through the

monooxygenases in yeast (Green and Large 1983). The resulting methylamine is then transported into the peroxisome for the final demethylation step catalysed by Amo1. The cytosolic Amo2 would therefore play little or no role in the demethylation of internally generated methylamine in Sc. stipitis due to spatial separation between internally generated methylamine in the endoplasmic reticulum and the cytosolic Amo2 enzyme (Fig. 5). This scenario would require the catabolism of choline, trimethylamine or dimethylamine is predominantly demethylated by Amo1. The intracellular localisation of the Cmol choline monooxygenase has not yet been established and its placement in the peroxisome in the current diagram should be considered entirely speculative (see text for details)

majority of methylamine to be transferred from the endoplasmic reticulum to Amo1 in the peroxisome in a manner that would largely bypass Amo2 the cytosol. A simpler explanation is that the $A M O 2$ gene is expressed at a lower level in Sc. stipitis when cultivated on dimethylamine, trimethylamine or choline as compared to cultivation on methylamine. Previous enzyme activity studies on $O$. boidinii cell extracts showed that Amo2 enzymatic activity was not 
detectable when cells had been cultivated on dimethylamine, trimethylamine or choline (Haywood and Large 1981), which would support such a hypothesis. Expression analyses of individual genes in Sc. stipitis were beyond the scope of the current study but may be pursued in future studies. The intracellular localisation of the choline monooxygenase $\mathrm{Cmo1}$ has not yet been established but the presence of a conserved C-terminus specific to fungal members of this protein family (Linder 2014) could indicate the presence of a noncanonical type 1 peroxisomal targeting signal (PTS1; Brocard and Hartig 2006). However, the current placement of Cmo1 in the peroxisome by the author as shown in Fig. 5 should be considered entirely speculative.

The second general observation is that budding yeasts appear to have at least one additional, as-yet unidentified enzyme capable of converting $S$-formylglutathione into glutathione (Fig. 3a). Weak but detectable growth in a $\Delta f g h l$ background has now been demonstrated both in Sc. stipitis (this study; Fig. 3b) and in the methylotrophic yeast $O$. boidinii (Yurimoto et al. 2003). The fact that SFA1 is still essential for growth on all tested methylated amines in Sc. stipitis (Fig. 3b) would argue against an entire pathway that is functionally redundant with the cyclic glutathione-dependent formaldehyde oxidation pathway in this yeast. However, this might not be the case in other yeasts such as $O$. boidinii where there is still detectable growth in a $\Delta s f a l$ background (Yurimoto et al. 2003).

The third and final observation is that the demethylation of di- and trimethylamine does not appear to involve the CYP protein family in Sc. stipitis. Whether or not this also the case in other budding yeasts remains to be established. Previous studies on the catabolism of secondary and tertiary amines have established that these enzymatic activities are localised to the endoplasmic reticulum in yeast and are sensitive to heme-binding inhibitors such carbon monoxide and cyanide (Green and Large 1983, 1984; Fattakhova et al. 1991). One possibility is that a non-CYP family heme-containing enzyme catalyses the demethylation of di- and trimethylamine. The author has so far been unsuccessful in identifying any other likely gene candidates based on sequence similarity and gene annotation data alone. A more straightforward strategy to identify the genes encoding the enzymes responsible for these demethylation reactions would be comparative expression analysis of Sc. stipitis cultivated on di- or trimethylamine versus a non-methylated reference nitrogen substrate. The author would like to add that to the best of his knowledge, the deletion mutants of PICST_49761 and PICST_63000 generated in the present study represents the first attempt at phenotypic characterisation of CYP families CYP5217 and CYP501 in yeast.

In conclusion, the yeast $S c$. stipitis has shown itself a promising system for the investigation of metabolic pathways not found in Sa. cerevisiae. The fairly recent development of genetic tools for targeted gene deletion in this species now enables more comprehensive reverse genetics studies in this yeast (Maassen et al. 2008; Linder 2014). As the present study demonstrates, there still remains much to be learned about the assimilation of alternative nitrogen sources in budding yeasts.

\section{Compliances with ethical standards}

Conflict of interest The author declares no conflict of interest.

Informed consent This article does not contain any studies with human participants or animals performed by the author.

Open Access This article is distributed under the terms of the Creative Commons Attribution 4.0 International License (http:// creativecommons.org/licenses/by/4.0/), which permits unrestricted use, distribution, and reproduction in any medium, provided you give appropriate credit to the original author(s) and the source, provide a link to the Creative Commons license, and indicate if changes were made.

\section{References}

Allen SJ, Holbrook JJ (1995) Isolation, sequence and overexpression of the gene encoding NAD-dependent formate dehydrogenase from the methylotrophic yeast Candida methylica. Gene 162:99-104

Briza P, Eckerstorfer M, Breitenbach M (1994) The sporulationspecific enzymes encoded by the DIT1 and DIT2 genes catalyze a two-step reaction leading to a soluble LL-dityrosine-containing precursor of the yeast spore wall. Proc Natl Acad Sci USA 91:4524-4528

Brocard C, Hartig A (2006) Peroxisome targeting signal 1: is it really a simple tripeptide? Biochim Biophys Acta 1763:1565-1573

Brouquisse R, Weigel P, Rhodes D, Yocum CF, Hanson AD (1989) Evidence for a ferredoxin-dependent choline monooxygenase from spinach chloroplast stroma. Plant Physiol 90:322-329 
Castresana J (2000) Selection of conserved blocks from multiple alignments for their use in phylogenetic analysis. Mol Biol Evol 17:540-552

Chen W, Lee MK, Jefcoate C, Kim SC, Chen F, Yu JH (2014) Fungal cytochrome $\mathrm{P} 450$ monooxygenases: their distribution, structure, functions, family expansion, and evolutionary origin. Genome Biol Evol 6:1620-1634

Degrassi G, Uotila L, Klima R, Venturi V (1999) Purification and properties of an esterase from the yeast Saccharomyces cerevisiae and identification of the encoding gene. Appl Environ Microbiol 65:3470-3472

Faber KN, Keizer-Gunnink I, Pluim D, Harder W, Ab G, Veenhuis M (1994) The N-terminus of amine oxidase of Hansenula polymorpha contains a peroxisomal targeting signal. FEBS Lett 357:115-120

Fattakhova AN, Ofitserov EN, Garusov AV (1991) Cytochrome P-450-dependent catabolism of triethanolamine in Rhodotorula mucilaginosa. Biodegradation 2:107-113

Green J, Large PJ (1983) Oxidation of dimethylamine and trimethylamine in methazotrophic yeasts by microsomal mono-oxygenases sensitive to carbon monoxide. Biochem Biophys Res Commun 113:900-907

Green J, Large PJ (1984) Subcellular localization and properties of partially purified dimethylamine and trimethylamine mono-oxygenase activities in Candida utilis. J Gen Microbiol 130:2577-2588

Green J, Haywood GW, Large PJ (1982) More than one amine oxidase is involved in the metabolism of primary amines supplied as nitrogen source. J Gen Microbiol 128:991-996

Haywood GW, Large PJ (1981) Microbial oxidation of amines. Distribution, purification and properties of two primaryamine oxidases from the yeast Candida boidinii grown on amines as sole nitrogen source. Biochem J 199:187-201

Huang FC, Peter A, Schwab W (2014) Expression and characterization of CYP52 genes involved in the biosynthesis of sophorolipid and alkane metabolism from Starmerella bombicola. Appl Environ Microbiol 80:766-776

Katoh K, Kuma K, Toh H, Miyata T (2005) mafft version 5: improvement in accuracy of multiple sequence alignment. Nucleic Acids Res 33:511-518

Kelly SL, Lamb DC, Corran AJ, Baldwin BC, Parks LW, Kelly DE (1995) Purification and reconstitution of activity of Saccharomyces cerevisiae P450 61, a sterol delta 22-desaturase. FEBS Lett 377:217-220

Large PJ (1986) Degradation of organic nitrogen compounds by yeasts. Yeast 2:1-34

Lee B, Yurimoto H, Sakai Y, Kato N (2002) Physiological role of the glutathione-dependent formaldehyde dehydrogenase in the methylotrophic yeast Candida boidinii. Microbiology 148:2697-2704

Linder T (2014) CMO1 encodes a putative choline monooxygenase and is required for the utilization of choline as the sole nitrogen source in the yeast Scheffersomyces stipitis (syn. Pichia stipitis). Microbiology 160:929-940

Maassen N, Freese S, Schruff B, Passoth V, Klinner U (2008) Nonhomologous end joining and homologous recombination DNA repair pathways in integration mutagenesis in the xylose-fermenting yeast Pichia stipitis. FEMS Yeast Res 8:735-743

Mori N, Shirakawa K, Uzura K, Kitamoto Y, Ichikawa Y (1988) Formation of ethylene-glycol and trimethylamine from choline by Candida tropicalis. FEMS Microbiol Lett 51:41-44

Ohkuma M, Muraoka S, Tanimoto T, Fujii M, Ohta A, Takagi M (1995) CYP52 (cytochrome P450alk) multigene family in Candida maltosa: identification and characterization of eight members. DNA Cell Biol 14:163-173

Sanglard D, Loper JC (1989) Characterization of the alkaneinducible cytochrome P450 (P450alk) gene from the yeast Candida tropicalis: identification of a new P450 gene family. Gene 76:121-136

Sasnauskas K, Jomantiene R, Januska A, Lebediene E, Lebedys J, Janulaitis A (1992) Cloning and analysis of a Candida maltosa gene which confers resistance to formaldehyde in Saccharomyces cerevisiae. Gene 122:207-211

Tamura K, Stecher G, Peterson D, Filipski A, Kumar S (2013) MEGA6: molecular Evolutionary Genetics Analysis version 6.0. Mol Biol Evol 30:2725-2729

Turi TG, Kalb VF, Loper JC (1991) Cytochrome P450 lanosterol 14 alpha-demethylase (ERG11) and manganese superoxide dismutase (SOD1) are adjacent genes in Saccharomyces cerevisiae. Yeast 7:627-630

van der Walt JP (1962) Utilization of ethylamine by yeasts. Antonie Van Leeuwenhoek 28:91-96

van Dijken JP, Bos P (1981) Utilization of amines by yeasts. Arch Microbiol 128:320-324

Warringer J, Ericson E, Fernandez L, Nerman O, Blomberg A (2003) High-resolution yeast phenomics resolves different physiological features in the saline response. Proc Natl Acad Sci USA 100:15724-15729

Yurimoto H, Lee B, Yano T, Sakai Y, Kato N (2003) Physiological role of $S$-formylglutathione hydrolase in $\mathrm{C}(1)$ metabolism of the methylotrophic yeast Candida boidinii. Microbiology 149:1971-1979

Zwart K, Veenhuis M, van Dijken JP, Harder W (1980) Development of amine oxidase-containing peroxisomes in yeasts during growth on glucose in the presence of methylamine as the sole source of nitrogen. Arch Microbiol 126:117-126

Zwart KB, Veenhuis M, Harder W (1983) Significance of yeast peroxisomes in the metabolism of choline and ethanolamine. Antonie Van Leeuwenhoek 49:369-385 